\title{
Evaluation and management of cases of infertility in a limited resource rural setup
}

\section{Jajoo S S ${ }^{1}$, Chandak $\mathrm{N} \mathrm{U}^{2}$}

\section{Abstract}

Background: Infertility is a global medical problem with important psychological, economical, social, marital and medical implications. Majority of the infertile couples live in resource-poor countries, with limited access to the sophisticated assisted reproductive techniques. Also high-quality, low-cost infertility clinics are not available in the public sector. This study is to evaluate infertile couples in a resource limited setting; in which giving maximum benefit and reducing the treatment costs were the major stand.

Aims:

- To evaluate the cases of infertility in rural area with available resources in order to find the etiology.

- To manage the cases of infertility in rural area with available resources.

Method: A total of hundred patients, including both primary and secondary infertility, were evaluated right from history and further investigations and interventions as and when required. A note was made of every visit so as to minimize the treatment time and cost of the couple.

Result: A conception rate of $19 \%$ was achieved with the available limited resources.

Conclusion: Stepwise management of an infertile couple with proper planning of all the visits and judicial use of the available resources would give maximum positive outcome and minimize the treatment cost to an appreciable extent.

Key words: infertility, rural setup, limited resources

\section{Introduction}

Infertility is defined as failure of a couple to conceive after 12 months of regular intercourse without the use of contraception in women less than 35 years of age; and after six months of regular intercourse without use of contraception in women 35 years and older ${ }^{1}$. Infertility is a common condition with important psychological, economical, social, marital and medical implications. The worldwide estimates of infertility range between 8 and 12 percent of the couples of reproductive-age group ${ }^{2}$.

\footnotetext{
${ }^{1}$ Professor,

${ }^{2}$ Resident, Department of Obstetrics and Gynaecology, Jawaharlal Nehru Medical College, Sawangi-Meghe, Wardha, Maharashtra, India.

Correspondence: N U Chandak

E-mail: dr.nehachandak@gmail.com
}

Although infertility is a global medical problem, affecting an estimated 60-80 million couples, the vast majority of them live in low resource countries ${ }^{3,4}$. Infertility is a menace to a couple, challenge to a physician and a prospect to a scientist; all of whom strive towards one goal - a healthy child. The infertile couple needs basic education, medical advice and frequent follow-ups and often access to expensive technology. Patients living in rural areas and those with low income find it difficult to seek healthcare for infertility related issues because of the treatment costs, long duration of therapy, frequent visits to the hospital and need to travel long distances for expensive interventions. Most infertile couples have poor access to Assisted Reproductive Technologies (ART) programmes because they are required to bare the cost. ART clinics are expensive to set up and have high recurring costs ${ }^{3}$. They require trained personnel, embryologists, biochemists, pathologists, sophisticated equipments, instruments, reagents, disposables etc. In addition, high quality, low-cost infertility clinics are not available in the public sector. Thus, infertility breeds social, financial, psychological and family problems, which neither infertile couples nor their doctors are able to cope with.

This study was designed to evaluate infertile couples in a resource limited setting; where a model was developed so as to reduce treatment costs - by ensuring that the basic fertility workup is done during the first visit including history-taking, physical examination and the simple first-hand investigations. Also the first visit was used to educate the couple and fulfill the emotional, informational and diagnostic needs of the patients. Finally, estimation of cost of evaluating and treating an infertile couple in a resource limited setting was done.

\section{Materials and methods}

From 2010 to 2012, out of all the infertile women anxious to conceive, attending the gynaecology outpatient department (OPD) for infertility investigations, 100 cases at random were considered for the purpose of the study.

Selection criteria: Following women were included in the study -

1. Cases of primary infertility

2. Cases of secondary infertility

Exclusion criteria: Following cases were excluded from the study -

1. Subjects who have undergone previous tubal surgeries, either tubal ligation or recanalisation

2. Patients not willing to participate in the study

\section{Collection of data:}

- The time period of the patient was tentatively planned and every first opportunity was held upright for gathering maximum positive information. 
- As the patient came to the OPD, a detailed history pertaining to infertility and other relevant history was asked and recorded, including the data if patient had previous treatment documents.

- In this first visit, the couple was explained in detail about the normal physiology and the fertile period.

- Then they were subjected to certain basic blood investigations, ultrasonography (USG) pelvis and husband's semen analysis (HSA); and were asked to followup with the next menstrual cycle.

- Meanwhile, the patient was treated for her complaints, if any.

- During the follow-up visit, all the previous investigation reports were noted and the ovulation / follicular study was advised from day 10 th of cycle.

- If she is a case of secondary infertility, in the same cycle hysterosalphingography (HSG) was advised at day 7 th or 8 th of the cycle. Whenever block was diagnosed on HSG, during same sitting fallopian tube canalization (FTC) was done with a guide wire and an oil based dye, flushed for better patency of tubes.

- Considering relative affordability of the patient and the need of further evaluation, they were subjected to diagnostic laparoscopy and / or hormonal assay.

- If laparoscopy was to be done, same sitting was utilized to do chromopertubation, drilling, fulguration, adhesiolysis etc. subject to requirement.

- Dilatation and curettage (D \& C) was done whenever laparoscopy was done and endometrial sample was sent for acid fast bacilli (AFB) stain, cytology and histopathology.

- Hormonal assay was considered when no apparent cause for infertility could be made with routinely available modalities.
- If male factor infertility was detected from husband semen analysis, antibiotics, supplementary drugs and if required clomiphene citrate were given to the male partner and those who had no results for 3 months were considered for intra-uterine insemination (IUI).

- Thus, maximum patient details were gathered in minimum visits.

More or less, every patient's etiology could be made out with these limited resources at our stake. Treatment was a simultaneous procedure.

\section{Results}

Of the 100 women (mean age $=27.73$ years; SD 4.5; range $=20$ to 40 years), $78(78 \%)$ presented with primary infertility while 22 (22\%) had secondary infertility. Women with secondary infertility tended to be older than those with primary infertility (30.5 vs. 26.9 years, $p=0.001)$. The age ranged from 22 to 39 years in women with primary infertility and that in case of secondary infertility it was 20 to 40 years.

Most patients with primary $(62.8 \%)$ as well as of secondary $(50.0 \%)$ infertility had duration of infertility between one and five years. The longest duration of infertility in primary infertility was observed to be 20 years and that in secondary infertility was 18 years.

\section{Figure 1. Distribution of cases due to female factor infertility according to different female factors}

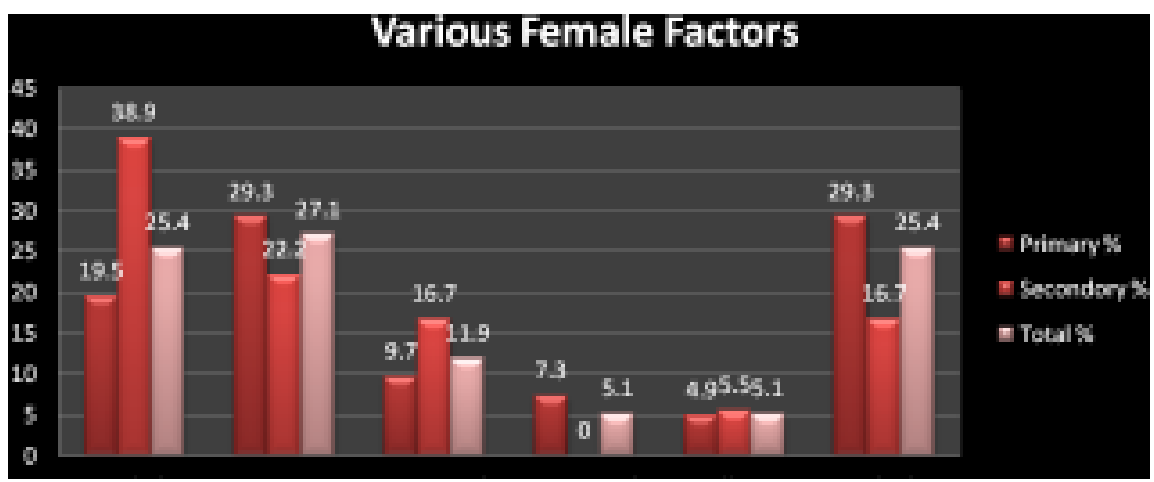

It was found that $71.8 \%$ cases of primary infertility had regular menstrual cycles, $21.4 \%$ had irregular cycles and 3.8\% cases had amenorrhea due to congenital uterine anomalies. In patients of secondary infertility, $63.6 \%$ patients had regular menstrual cycles, $27.3 \%$ had irregular menses and $9.1 \%$ cases were of ammenorrhoea (hyperprolactinaemia: 1 patient; Asherman's syndrome: 1 patient). In all, $76 \%$ cases had normal amount of menstrual flow, $16 \%$ had scanty flow, and $3 \%$ had menorrhagia due to fibroid and endometrial hyperplasia.

Female factor accounted for nearly half the cases of infertility (46\%) while the male factor $(30 \%)$ contributed to close to one third of the total cases. About $13 \%$ cases had combined cause and about $11 \%$ cases had unexplained infertility.

The various female factors responsible for infertility are as shown in Figure 1. In general, it was observed that majority $(27.1 \%)$ of cases had ovarian factor infertility. Among the primary infertility cases, ovarian factor was the commonest $(29.3 \%)$, whereas, tubal factor was found to be the commonest cause in secondary infertility cases (38.9\%). Hyperprolactinaemia was the leading hormonal cause of infertility and in few cases, it coexisted with hypothyroidism. Elevated levels of prolactin were found in $9.7 \%$ cases of primary infertility and $16.7 \%$ 
cases of secondary infertility. In about $5 \%$ patients we found association of infertility with factors such as cervical abnormalities, peritoneal adhesions, infections, and endometriosis. The category of 'multiple' included patients having more than one causative factor for infertility. A total of $29.3 \%$ patients with primary infertility and $16.7 \%$ patients with secondary infertility were found to belong to this category.

Of the 100 infertile couples, six men did not agree to undergo semen analysis. Of the 94 cases, who underwent semen analysis, 51 had normal semen analysis and 43 cases were found to have abnormal findings as depicted in Figure 2.

A baseline USG was done in the women and was found to be normal in 71 of 100 women; the rest had diverse abnormalities that ranged from ovarian (17 of 100) to uterine lesions (4 of 100).

A total of 93 patients underwent ovulatory study, out of which, $64 \%$ patients had ovulatory cycles and $29 \%$ had anovulatory ones. We did not do follicular study in those with congenital abnormalities; some patients did not turn up for the study.

\section{Figure 2. Distribution of cases according to abnormal semen findings}

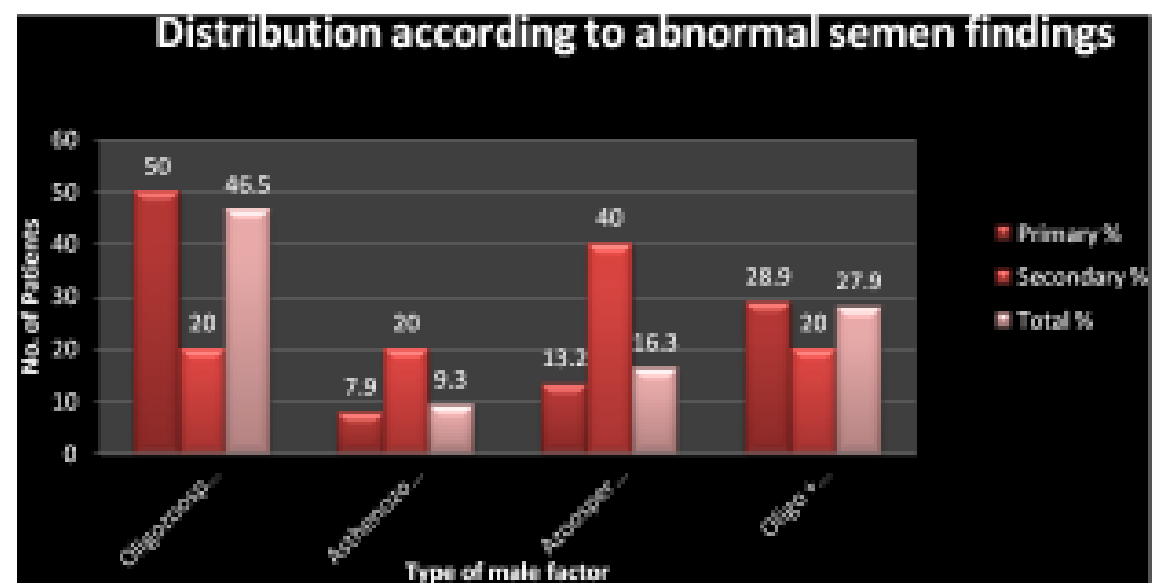

Figure 3. Distribution of cases which conceived, according to the different interventions made

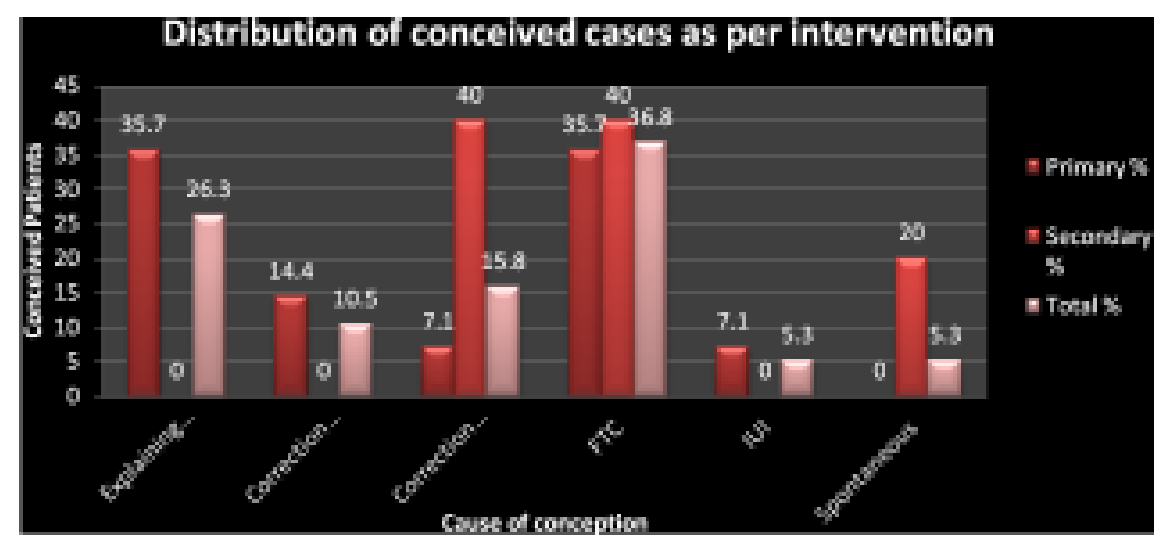

Diagnostic laparoscopy was undertaken in 38 cases and intervened whenever required. A total of 14 women had normal studies.

Table 1 shows various modalities used for treating the cases of infertility and their successful outcome. Out of all, the conception with fallopian tube canalization was statistically significant. A total of 19 cases $(19 \%)$ had conceived: 14 of $78(17.9 \%)$ patients with primary infertility and 5 of 22 $(22.7 \%)$ patients with secondary infertility.

Figure 3 gives the distribution of conceived cases as per the intervention done. Educating the couple by making them understand the normal physiology and the fertile period; and fallopian tube canalization were observed to be the two most successful methods of intervention $(26.3 \%$ and $36.8 \%$ conception rate respectively). One patient with secondary infertility spontaneously conceived (20\%).

In general, women tended to conceive early if they were young; the chance of a successful conception begins to fall as the women grew older. Thus, there was a correlation between age and infertility.

It was also observed that the chances of successful conception were highest $(68 \%)$ in women presenting with infertility within 1 to 5 years of marriage. The chances grew slim with the passage of time.

We also calculated the cost required to assess and treat each infertile woman. The mean cost of treatment was Rs 4584 (SD 1397; range: Rs 1600 to Rs 8900). The median cost of the treatment was Rs 4500 (IQR 3450 to 5600). As many as 61 patients could be treated in less than Rs 5000. The cost of treatment was almost similar in women with primary infertility compared to those with secondary infertility (Rs. 4639 vs. Rs 4386; $\mathrm{p}=0.22$ ). The difference in the cost was not statistically significant (Figure 4). 
Table 1. Distribution of cases according to treatment modality

\begin{tabular}{|c|c|c|c|c|c|c|c|}
\hline \multirow[t]{2}{*}{ Type of $t / t$} & \multicolumn{2}{|c|}{ Primary } & \multicolumn{2}{|c|}{ Secondary } & \multicolumn{2}{|c|}{ Total } & \multirow{2}{*}{$\begin{array}{l}\text { Z-value } \\
(\mathrm{Se} \geq 1.96)\end{array}$} \\
\hline & Received & Conceived & Received & Conceived & Received & Conceived & \\
\hline Ovulation induction & 20 & $2(10.00 \%)$ & 05 & $0(00.00 \%)$ & 25 & $2(08.00 \%)$ & $1.47 ; \mathrm{NS}$ \\
\hline Hormonal t/t & 12 & $1(08.33 \%)$ & 05 & $2(40.00 \%)$ & 17 & $3(17.65 \%)$ & $1.91 ; N S$ \\
\hline FTC & 15 & $5(33.33 \%)$ & 10 & $2(20.00 \%)$ & 25 & $7(28.00 \%)$ & $3.12 ; \mathbf{S}$ \\
\hline$D \& C$ & 33 & $0(00.00 \%)$ & 06 & $0(00.00 \%)$ & 39 & $0(00.00 \%)$ & NA \\
\hline Lap Drilling & 11 & $0(00.00 \%)$ & 01 & $0(00.00 \%)$ & 12 & $0(00.00 \%)$ & NA \\
\hline Lap Chromo-pertubation & 20 & $0(00.00 \%)$ & 04 & $0(00.00 \%)$ & 24 & $0(00.00 \%)$ & NA \\
\hline Lap Adhesiolysis & 04 & $0(00.00 \%)$ & 01 & $0(00.00 \%)$ & 05 & $0(00.00 \%)$ & NA \\
\hline IUI & 40 & $1(02.5 \%)$ & 05 & $0(00.00 \%)$ & 45 & $1(03.70 \%)$ & $1.01 ; \mathrm{NS}$ \\
\hline
\end{tabular}

Figure 4. Cost of workup in women with infertility

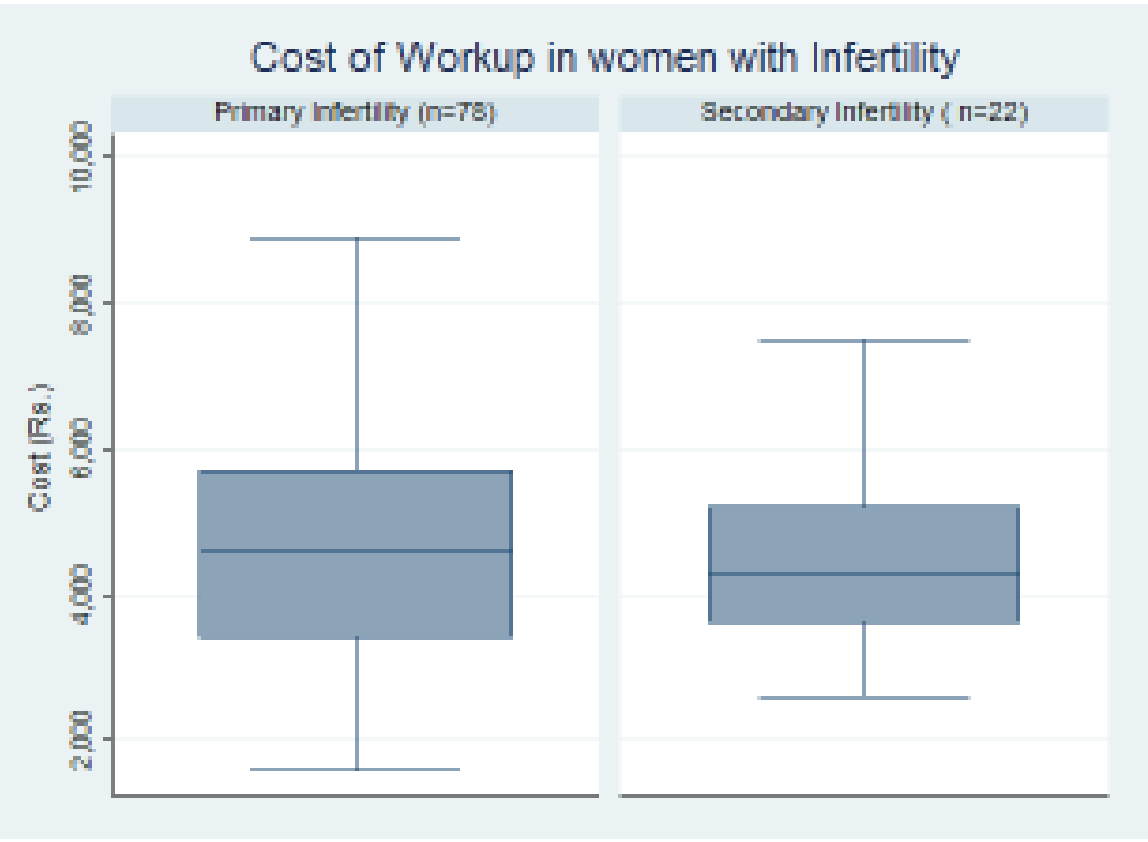

\section{Discussion}

We found that a total of 360 of 5534 $(6.5 \%)$ patients presenting to the ObGy OPD of our Institute had infertility; comparable with the prevalence reported by Abbas et al (2009) 5 (5.5\%).

In the present study, 78 of 100 infertile cases had primary infertility while $22 \%$ cases had secondary. Comparable results were found in certain studies as that of Shamila $S$ et al $(2011)^{6}$ - primary $82.48 \%$, secondary $17.52 \%$; Ikechebelu JI et al $(2003)^{7}-$ primary $77.2 \%$, secondary $22.8 \%$. Estimates of infertility prevalence have been shown to vary across regions within a country; thus, our findings add to the limited data on infertility from different regions of rural India.

In our study, the mean age of primary infertility was 26.9 years, while that of secondary infertility was 30.5 years. The overall mean age (SD) was $27.7 \pm 4.5$ years (range: 20 to 40 years) which was comparable to the study of Kamath et al (2010) ${ }^{8}$, in which the mean female age was $30.04 \pm 4.3$ years (range: 20-43 years). Women who conceived $(n=19)$ as old as those who did not $(\mathrm{n}=81)$ (28.1 years vs. 27.5 years; $\mathrm{p}=0.85)$.

In our study, female factor accounted for nearly half the cases of infertility (46\%) while the male factor (30\%) contributed to close to one third of the total cases. About $13 \%$ of cases had combined causes and about 11\% cases had unexplained infertility. Our numbers are in line with those reported previously by Shamila $S$ et al $(2011)^{6}$ - male factor $54.3 \%$, female factor 45.6\%; Abbas et al (2009) - male factor $25.3 \%$, female factor $57.5 \%$, combined $8 \%$, unexplained $16 \%$; Ikechebelu JI et al $(2003)^{7}$ - male factor $42.4 \%$, female factor $21.5 \%$, combined $20.7 \%$, unex-plained $11.1 \%$. 
We found that ovarian factor $(29.3 \%)$ was the commonest female factor responsible for primary infertility and that tubal factor (38.9\%) was the commonest in secondary infertility. In a study by Patel M et al (2012) tubal factor accounted for $29 \%$, ovarian for $36 \%$ and miscellaneous for $14 \%$. Another study by Abbas et al $(2009)^{5}$, showed tubal factor $46.6 \%$ and ovarian $58.8 \%$. Ovarian factors account for most cases of female infertility worldwide. As shown previously, we too found that most women with infertility had ovarian factors.

The overall observation of the semen findings in the present study is that $46.5 \%$ showed oligozoospermia, 9.3\% asthenozoospermia, $16.3 \%$ azoospermia and $27.9 \%$ showed oligo+asthenozoospermia. These results were in comparison with the studies of Patel M et al (2012) ${ }^{9}$ and Abbas et al (2009) ${ }^{5}$ as follows:

\begin{tabular}{lcc}
\hline $\begin{array}{l}\text { Abnormal sperm } \\
\text { parameters }\end{array}$ & $\begin{array}{c}\text { Patel et al } \\
\text { (2012) }\end{array}$ & $\begin{array}{c}\text { Abbas et al } \\
\text { (2009) }\end{array}$ \\
Oligozoospermia & $23.5 \%$ & - \\
Asthenozoospermia & $17.4 \%$ & $15.5 \%$ \\
Azoospermia & $50 \%$ & $20.7 \%$ \\
$\begin{array}{l}\text { Oligo + } \\
\text { asthenozoospermia }\end{array}$ & - & $63.8 \%$ \\
\hline
\end{tabular}

During their reproductive lives, 10 to $15 \%$ of couples are unable to achieve conception and deliver a living child after 1 year of unprotected coitus $^{10}$. The proportion of women unable to bear children increases with age. Our data shows that most women $(60 \%)$ presented with infertility within 1 to 5 years of marriage; while only $4 \%$ women showed up after 16 years of marriage. This observation is in line with a previous study by Shamila $S$ et al $(2011)^{6}$ in which $51.8 \%$ cases were infertile for 5 years and only less than $1 \%$ cases presented with infertility after 25 years.

The age of the female also had an effect on conception. Women tended to conceive more if they were aged less than 30 years old. Beyond 30 years of age, the rate of conception declines. In our study, women who conceived had a similar duration of infertility compared to those who did not (5.28 years vs. $5.86 ; p=0.60$ ). Our results are in agreement with those reported by Fretts RC (2012) ${ }^{11}$ as follows:

\begin{tabular}{ccc}
\hline $\begin{array}{c}\text { Age groups } \\
\text { (years) }\end{array}$ & $\begin{array}{c}\text { Fretts } R C \\
\text { (2012) }\end{array}$ & $\begin{array}{c}\text { Present } \\
\text { study }\end{array}$ \\
$<31$ & $74 \%$ & $73.6 \%$ \\
$31-35$ & $62 \%$ & $21.1 \%$ \\
$>35$ & $54 \%$ & $5.3 \%$ \\
\hline
\end{tabular}

Diagnostic evaluation for infertility in women should be conducted in a systematic, expeditious, and costeffective manner to identify all relevant factors with initial emphasis on the least invasive methods for detection of the most common causes of infertility. We followed this approach and explored the best way to treat infertile couples in the most cost effective manner. Overall, an infertile couple incurred the mean cost Rs. 4584 (SD 1397; range: Rs. 1600 to Rs. 8900) that included visits to the hospital, assessment and planning, diagnostics, interventions, follow-up, monitoring and inpatient hospitalisation. We could offer high value; low-cost therapy to most patients: as many as 61 patients could be treated in less than Rs 5000. The cost of treatment did not differ between those who conceived compared to those who did not (Rs. 4642 vs. 4617; $\mathrm{p}=0.94)$. This contrasts with several studies which reported a very high recurring treatment $\operatorname{cost}^{12}$. Although ART has transformed the treatment of infertility and also its diagnosis, patients living in rural areas and urban slums cannot even think of accessing ART - given the expenses involved. Also, ART are sometimes used to treat incompletely-evaluated patients; women are recommended to proceed to ART following an accelerated and often incomplete work-up. We believe that care must be taken to avoid exploitation of the infertile couple with expensive, unnecessary tests, procedures, and treatments. Performing a battery of comprehensive investigations may explain or define causes of otherwise unexplained infertility, but when many tests are carried out as a complete package for evaluation of an infertile couple, then the false-positive results can increase the subsequent cost of the treatment exponentially. We used the traditional approach to assess and manage our infertile couples with the most basic tools starting from history taking to IUI.

We used laparoscopy selectively, limiting it to 36 of 100 patients and found lesions which could be addressed. Our results suggest that if gynecologists follow a structured approach, even in resource setting, as many as $19 \%$ women could conceive without incurring a large amount of cost for the success.

Finally, we explored the feasibility of using a pragmatic, low-cost, highvalue approach to asses and treat couples presenting with primary and secondary infertility in a resource limited setting. We found that the prevalence of infertility was about $6.5 \%$ in our setting and primary infertility outnumbered secondary infertility by a factor of four. Female factors accounted for half the causes, male factors accounted for about a third of causes. One out of 5 females could conceive in our setting.

\section{Conclusion}

Infertility is a significant problem in developing countries and women bear the major brunt of this devastating social, medical and economical burden, though men as well, hold a prominent share. Although this problem is now known to be global, the vast majority is rural and financially deprived. Tackling infertility, focus needs to be on proper utilization of the available resources at our stake and the management of time.

Right away, the first visit has to be encashed for detail history and clinical examination, baseline pelvic USG, semen analysis and other blood 
investigations; with simultaneous assurance to the couple by means of counseling and tentative management plan. For sure, such an approach will take us to some or other diagnosis and thus the management can be streamlined during the second visit, in the form of hormonal assay, hysterosalphinsography with or without fallopian tube canalization (FTC) or IUI as per the requirement.

In our study, the most effective modality of treatment giving positive outcome was found out to be FTC (28\%) where blocked tubes were diagnosed and intervened in the same sitting.

Minimizing the treatment time will ultimately minimize the cost of treatment with better outcome. In our study, as many as 61 patients could be treated in less than Rs. 5000/-, the mean cost of treatment being Rs. 4584/-.

Despite all these measures, there could be few cases where Artificial Reproductive Technologies need to be considered and for them, collaboration of public and private sectors may yield accessible low cost treatment with resource limited settings.

\section{Acknowledgements}

We are overwhelmed by our families' support during this study. We are grateful to our fellow colleagues and the colleagues in the departments of radiology, anaesthesiology and pathology; the paramedical staff, technicians and all others who were directly or indirectly involved. Last but not the least, we thank all our patients for their faith in us.

\section{REFERENCES}

1. Practice Committee of the American Society for Reproductive Medicine. Definition of infertility and recurrent pregnancy loss. Fertil Steril 2008; 90: 560.

2. World Health Organisation. Infertility: a tabulation of available data on prevalence of primary and secondary infertility. 1991. WHO: Geneva. Programme on Maternal and Child Health and Family Planning, Division of Family Health 1991.

3. Sharma S, Mittal S, Aggarwal P. Management of infertility in low resource countries. BJOG 2009; 116 (Suppl. 1): 77-83.

4. Widge A, Cleland J. The public sector's role in infertility management in India. Health Policy and Planning 2009:1-8.
5. Abbas A, Seyedhassani SM, Nasim T. The epidemiological and etiological aspects of infertility in Yazd province if Iran. Indian J Repro Med. 2009; 7(3):117-22.

6. Shamila S, Sasikala SL. Primary report on the risk factors affecting in South Indian districts of Tamilnadu and Kerala. Indian J Comm med. 2011; 36(1).

7. Ikechebelu JI, Adinma JI, Orie EF, Ikegwuonu SOJ. High prevalence of male infertility in southeastern Nigeria. J Obstet Gynaecol. 2003 Nov; 23(6): 657-9.

8. Kamath MS, Bhave P, Aleyamma TK, Nair R, Chandy A, Mangalaraj AM et al. Predictive factors for pregnancy after intrauterine insemination: A prospective study of factors affecting outcome. J Hum Reprod Sci 2010; 3: 129-34.

9. Patel M, Jain S et al. Prevalence of different factors responsible for infertility. Res $\mathrm{J}$ Recent Sci. 2012; 1.

10. Berek J, editor. Berek and Novak's gynaecology (ed 15th).

11. Fretts FC. Effects of advanced age on fertility and pregnancy in women. Wolters Kluwer Health. 2012 Sept (Suppl.).

12. Case AM. Infertility evaluation and management strategies for family physicians. Can Fam Physician 2003;49: 1465-72. 